\title{
Antenna Radiation Efficiency Estimation From Backscattering Measurement Performed Within Reverberation Chambers
}

\author{
Wafa Krouka, François Sarrazin, Member, IEEE, Julien de Rosny, Senior Member, IEEE, Adnane Labdouni, \\ and Elodie Richalot, Member, IEEE
}

\begin{abstract}
This paper presents a contactless measurement method for antenna radiation efficiency estimation within reverberation chambers (RCs). This method does not require to connect any analyzer to the antenna under test (AUT). The AUT radiation efficiency is obtained from the differential measurement of the RC composite $Q$-factor estimated for two AUT load conditions, namely an open circuit and a $50 \Omega$ load. Remotelycontrolled RF switches are used in order to keep the setup identical for both measurements. The method is experimentally validated in two RCs of different volumes $\left(1 \mathrm{~m}^{3}\right.$ and $\left.19 \mathrm{~m}^{3}\right)$ with a wideband patch antenna in the $1.8 \mathrm{GHz}$ to $2.8 \mathrm{GHz}$ frequency range. The estimated efficiency is found to be in very good agreement with the one obtained through a conventional invasive RC measurement technique. This new method is suitable for miniature and buried antenna characterization without access to its port.
\end{abstract}

Index Terms-Antenna radiation efficiency, backscattering measurement, contactless measurement, quality factor, reverberation chamber.

\section{INTRODUCTION}

A NTENNA radiation efficiency is defined as the ratio between the radiated power and the incident power, i.e., the one that goes through the antenna port [1]. Its measurement has been initially performed using a transmission-type setup within an anechoic chamber (AC) by applying the gainintegration technique. Since first studies in 2001 [2], reverberation chambers (RCs) became very attractive to measure the antenna radiation efficiency [3]-[6]. Indeed, thanks to the statistically isotropic and homogeneous field within the RC working volume, such measurement neither exhibits alignment constraints nor require the antenna under test (AUT) rotation. Moreover, the use of a reference antenna can be avoided by comparing the $\mathrm{RC} Q$-factor estimated in the time and frequency domains [7], [8]. However, all current methods require an invasive setup, i.e., a setup where the AUT needs to be connected to the analyzer implying connectors and long cables.

The presence of cables in the measurement setup leads to unwanted interactions between the AUT and the cables [9], [10]. Two different phenomena occur: 1) the feed cables are placed in the antenna near-field zone; therefore both resistive

W. Krouka, F. Sarrazin, A. Labdouni and E. Richalot are with the ESYCOM lab, Univ Gustave Eiffel, CNRS, F-77454 Marne-la-Valle, France (e-mail: francois.sarrazin@univ-eiffel.fr).

J. de Rosny is with ESPCI Paris, PSL University, CNRS, Institut Langevin, Paris, France (julien.derosny@espci.fr). and reactive parts of the input impedance are modified, as well as the antenna radiation characteristics, and 2) The imperfect balance between the radiating element and the ground plane causes current leaking on the outer shielding of the feed cables, so that the cables themselves act as radiators [11]-[13]. These two effects become critical when dealing with electrically small antennas and lead to inaccurate results [14], [15].

Different ways have been investigated to overcome this issue in an AC: limiting the cable effects by adding ferrites, quarter-wavelength sleeves [17] or balun [16]; compensating for disturbancies introduced by the measurement setup thanks to prior electromagnetic simulations [14] or post-processing [18] ; replacing the coaxial cables by optical links [19], [20]; performing antenna backscattering measurement. The latter is of particular interest as it enables a contactless measurement, i.e., without the need to connect the AUT to an analyzer. It is based on several measurements with either a varying load [21] or a few discrete ones [22]. First introduced in an AC, such techniques have been recently applied within an RC [23]-[25] but only for antenna radiation pattern estimation.

The insertion of lossy objects within an $\mathrm{RC}$ has been studied in terms of absorption [26] and diffusion [27] whereas the specific case of antennas has been first dealt with as a dissipative load impedance [28] before that a refined model was proposed recently [29] in order to take into account the reflections due to the load impedance mismatch. In this paper, we take benefit from these studies to introduce an original contactless measurement technique that allows retrieving the antenna radiation efficiency.

This paper is organized as follows: Section III introduces the theoretical background for the contactless measurement method. Section III describes the experimental setup within an $\mathrm{RC}$ as well as the radiation efficiency results obtained using the contactless method and compared to conventional measurements. The very good agreement between the two methods is confirmed by a second measurement in a smaller $\mathrm{RC}$ in Section IV. Finally, a conclusion ends this paper.

\section{THEORY}

The RC $Q$-factor is proportional to the ratio between the stored energy $U$ and the dissipated power $P_{\mathrm{d}}$ within the cavity as:

$$
Q=\frac{\omega U}{P_{\mathrm{d}}} .
$$


Let us consider an RC measurement setup where two arbitrary antennas are connected to a VNA in order to measure the RC $Q$-factor (from transmission coefficient). $N$ identical AUTs are located on masts within the working volume and unconnected to any measurement device. In this configuration, the RC $Q$-factor $Q_{\mathrm{L} x}$ can be decomposed as

$$
Q_{\mathrm{L} x}^{-1}=Q_{\mathrm{c}}^{-1}+N Q_{\mathrm{a}, \mathrm{L} x}^{-1}
$$

where $Q_{\mathrm{a}, \mathrm{L} x}$ is associated with the losses of a single AUT terminated by a load impedance $Z_{\mathrm{L} x}$ and $Q_{\mathrm{c}}$ is related to all other sources of losses (wall, transmitting and receiving antennas, stirrer, masts.). In order to isolate $Q_{\mathrm{a}, \mathrm{L} x}$, one could perform an "empty" measurement where the $N$ AUTs are removed from the RC. Indeed, the difference between the $Q$-factors for the two measurements (with and without AUTs) leads to $N Q_{\mathrm{a}, \mathrm{L} x}$. However, this requires two distinct measurements whose setup will eventually vary due to manual handling to remove the $N$ AUTs. This effect will be highlighted in section IV] Thus, we suggest in this paper to perform two measurements, both with the $N$ AUTs located within the RC, but terminated by two different load impedances $Z_{\mathrm{L} 1}$ and $Z_{\mathrm{L} 2}$. The loads are modified thanks to remotely controlled switches and are identical for all AUTs. Hence, no manual handling is performed in this case. From (2), the difference between the $Q$-factors measured for two load impedances can be expressed as:

$$
Q_{\mathrm{L} 1}^{-1}-Q_{\mathrm{L} 2}^{-1}=N\left(Q_{\mathrm{a}, \mathrm{L} 1}^{-1}-Q_{\mathrm{a}, \mathrm{L} 2}^{-1}\right)
$$

The term $Q_{\mathrm{c}}$ vanishes in this difference as the setup remains identical. A first expression of the antenna $Q$-factor within an $\operatorname{RC} Q_{\mathrm{a}}$, referred thereafter as eq 1 , has been derived by Hill et al. in [28] such as:

$$
Q_{\mathrm{a}, \mathrm{L} x}^{\mathrm{eq} 1}=\frac{Q_{\mathrm{a}}^{0}}{\eta_{\mathrm{a}}\left(1-\left|\Gamma_{\mathrm{a}, \mathrm{L} x}\right|^{2}\right)}
$$

where $Q_{\mathrm{a}}^{0}=16 \pi^{2} V / \lambda^{3}$ is the $Q$-factor of an ideal lossless antenna, and $\Gamma_{\mathrm{a}, \mathrm{L} x}$ is the reflection coefficient between the AUT input impedance and its load impedance $Z_{\mathrm{L} x} . \Gamma_{\mathrm{a}, \mathrm{L} x}$ can be computed as [32]:

$$
\left|\Gamma_{\mathrm{a}, \mathrm{L} x}\right|=\left|\frac{\Gamma_{\mathrm{L} x}-\Gamma_{\mathrm{a}}^{\dagger}}{1-\Gamma_{\mathrm{L} x} \Gamma_{\mathrm{a}}}\right|
$$

where $\Gamma_{\mathrm{a}}$ and $\Gamma_{\mathrm{L} x}$ are the reflection coefficients of the AUT and the load impedance, respectively, according to $50 \Omega$. The superscript ${ }^{\dagger}$ stands for the complex conjugate. In 2018, Cozza [29] suggested modifying the $Q$-factor formula (4) in order to take into account the power reflected by the antenna load which is re-radiated towards the RC. The modified formula, referred then as eq2, is given as:

$$
Q_{\mathrm{a}, \mathrm{L} x}^{\mathrm{eq} 2}=\frac{Q_{\mathrm{a}}^{0}}{1-\eta_{\mathrm{a}}^{2}\left|\Gamma_{\mathrm{a}, \mathrm{L} x}\right|^{2}}
$$

Replacing $Q_{\mathrm{a}, \mathrm{L} 1}$ and $Q_{\mathrm{a}, \mathrm{L} 2}$ in $(3)$ by their expressions from (4) and (6), the AUT radiation efficiency $\eta_{\mathrm{a}}$ can be retrieved through two relations as:

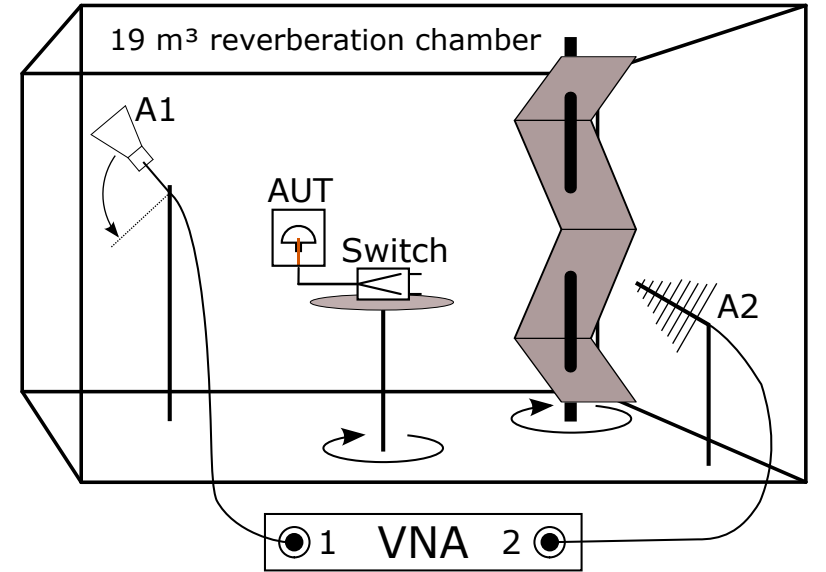

Fig. 1. Antenna efficiency measurement setup in the ESYCOM RC. A1 and $\mathrm{A} 2$ are a horn antenna and a log-periodic antenna, respectively, and are connected to a VNA. A1 is oriented towards an edge of the RC and 17 different positions are considered. A2 is oriented towards the mode stirrer. The vertical mode stirrer rotates around its axis and 72 positions are considered over a revolution. The AUT is located on a mast and connected to a mechanical switch through a $30-\mathrm{cm}$ cable. Four different orientations are considered for the AUT.

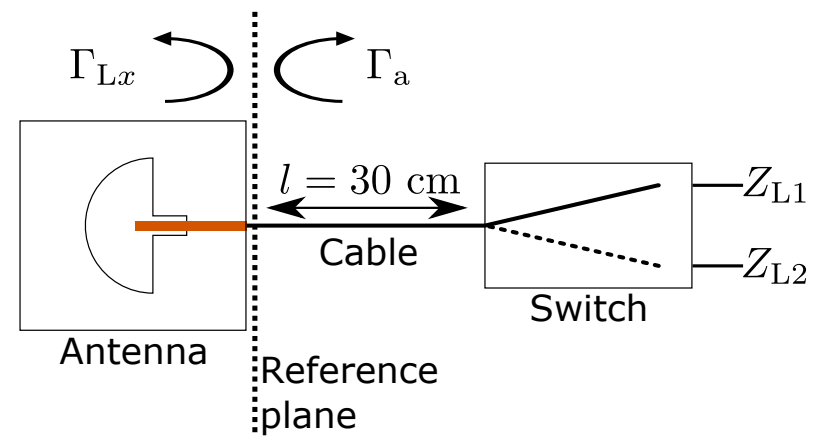

Fig. 2. Reference plane considered for measuring both the antenna and the load reflection coefficients.

$$
\eta_{\mathrm{a}}^{\mathrm{eq} 1}=\frac{Q_{\mathrm{a}}^{0}\left(Q_{\mathrm{L} 1}^{-1}-Q_{\mathrm{L} 2}^{-1}\right)}{N\left(\left|\Gamma_{\mathrm{a}, \mathrm{L} 2}\right|^{2}-\left|\Gamma_{\mathrm{a}, \mathrm{L} 1}\right|^{2}\right)}
$$

and

$$
\eta_{\mathrm{a}}^{\mathrm{eq} 2}=\sqrt{\frac{Q_{\mathrm{a}}^{0}\left(Q_{\mathrm{L} 1}^{-1}-Q_{\mathrm{L} 2}^{-1}\right)}{N\left(\left|\Gamma_{\mathrm{a}, \mathrm{L} 2}\right|^{2}-\left|\Gamma_{\mathrm{a}, \mathrm{L} 1}\right|^{2}\right)}} .
$$

It can be noticed that $\eta_{\mathrm{a}}^{\mathrm{eq} 2}$ is the square root of $\eta_{\mathrm{a}}^{\mathrm{eq} 1}$. The radiation efficiency estimation $\eta_{\mathrm{a}}^{\text {eq2 }}$ using $(8)$ is expected to be more accurate as it is based on the revised $Q$-factor formula from [29], but the estimation $\eta_{\mathrm{a}}^{\text {eq1 }}$ using (7) will be evaluated in the following sections in order to highlight the limitations of the previous model from [28].

\section{MeAsurement}

\section{A. Experiment}

The setup is presented in Fig. 1. This experiment has been conducted at the ESYCOM laboratory in an RC whose dimensions are $2.95 \mathrm{~m} \times 2.75 \mathrm{~m} \times 2.35 \mathrm{~m}$, equipped with 


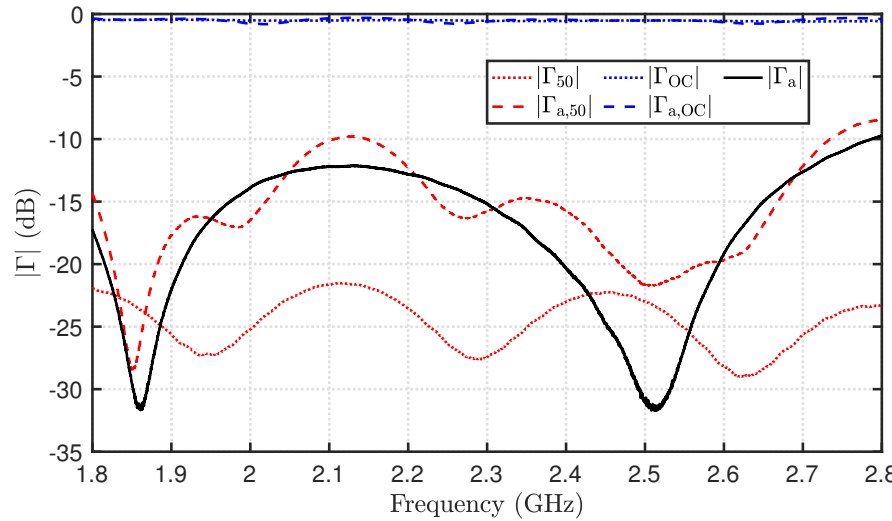

Fig. 3. AUT and load impedances reflection coefficients as a function of frequency.

a rotating metallic mode stirrer (72 equally spaced positions are considered). Two antennas (A1 and A2) are connected to a Rohde\&Schwarz ZNB20 vector network analyzer (VNA) in order to measure their scattering parameters in the $1.8 \mathrm{GHz}$ to $2.8 \mathrm{GHz}$ frequency range (10001 frequency points). $\mathrm{A} 1$ is oriented towards an edge of the $\mathrm{RC}$ whereas $\mathrm{A} 2$ is oriented towards the mode stirrer. A1 is positioned on a verticallyrotating mast in order to perform source stirring over 17 positions. Both antennas are carefully positioned to avoid any direct coupling between them for all configurations; indeed, A1 and A2 are orthogonally positioned and polarized. The AUT is a wideband slot-based patch antenna (details in [8]) that is connected, through a $30-\mathrm{cm}$ long coaxial cable, to a remotely-controlled mechanical switch that allows switching between two load impedances (Fig. 2). The AUT and the switch are positioned on a rotating mast and four positions are considered to perform the AUT stirring. In order to maximize the difference between the two measured $Q$-factors, and thus, the measurement sensitivity, an open circuit (OC) and a $50 \Omega$ load are chosen as the two load impedances $Z_{\mathrm{L} 1}$ and $Z_{\mathrm{L} 2}$, respectively. All measurements are performed in a single run, without manual manipulation.

To assess the validity of (7) and (8), we first perform an invasive estimation of $\left|\Gamma_{\mathrm{a}, \mathrm{L} x}\right|$. With a VNA, we measure on the one hand the AUT reflection coefficient $\Gamma_{\mathrm{a}}$ within an AC, and on the other hand the reflection coefficients $\Gamma_{\mathrm{L} 1}=\Gamma_{\mathrm{OC}}$ and $\Gamma_{\mathrm{L} 2}=\Gamma_{50}$, associated to the two load impedances $Z_{\mathrm{L} 1}$ and $Z_{\mathrm{L} 2}$. As shown in Fig. 2, the measurement reference plane is located at the antenna's port so that the losses associated to the cable and the switch are included in $\Gamma_{\mathrm{OC}}$ and $\Gamma_{50}$. From this 2-step measurement, $\left|\Gamma_{\mathrm{a}, \mathrm{OC}}\right|$ and $\left|\Gamma_{\mathrm{a}, 50}\right|$ are deduced from (5) and results are presented in Fig. 3 as a function of the frequency. We can notice that the AUT is well matched to $50 \Omega$ in the whole frequency range $\left(\left|\Gamma_{a}\right|\right.$ less than $\left.-10 \mathrm{~dB}\right)$. Due to the switch insertion losses as well as the losses due to the cable between the AUT and the switch, $\left|\Gamma_{\mathrm{OC}}\right|$ is not equal to $0 \mathrm{~dB}$ but its average over the frequency band is of $-0.51 \mathrm{~dB} .\left|\Gamma_{50}\right|$ is less than $-20 \mathrm{~dB}$; thus, this load acts as a good matched load even though the presence of the switch and the cable. As the AUT is well matched, $\left|\Gamma_{\mathrm{a}, 50}\right|$ and $\left|\Gamma_{\mathrm{a}, \mathrm{OC}}\right|$ exhibit similar behaviors to $\left|\Gamma_{\mathrm{a}}\right|$ and $\left|\Gamma_{\mathrm{OC}}\right|$, respectively.

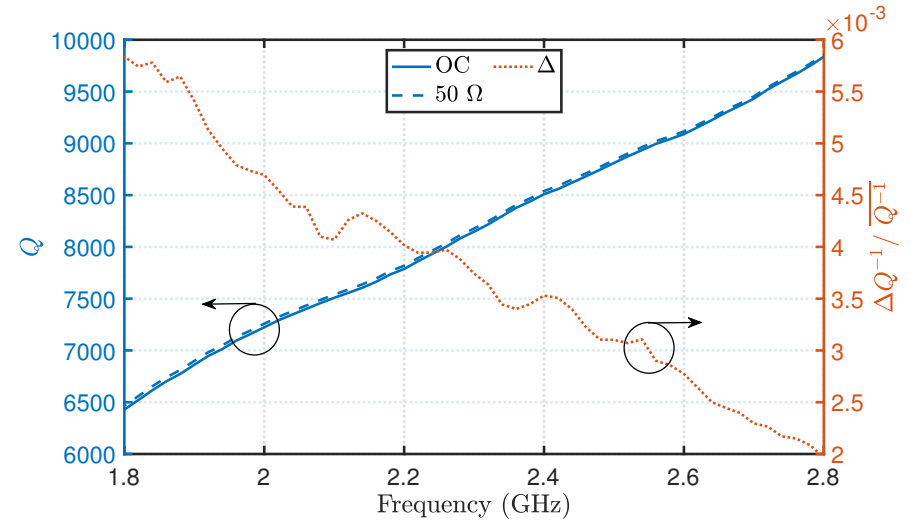

Fig. 4. $Q$ as a function of frequency (left) and $\Delta Q^{-1} / \overline{Q^{-1}}$ as a function of frequency (right).

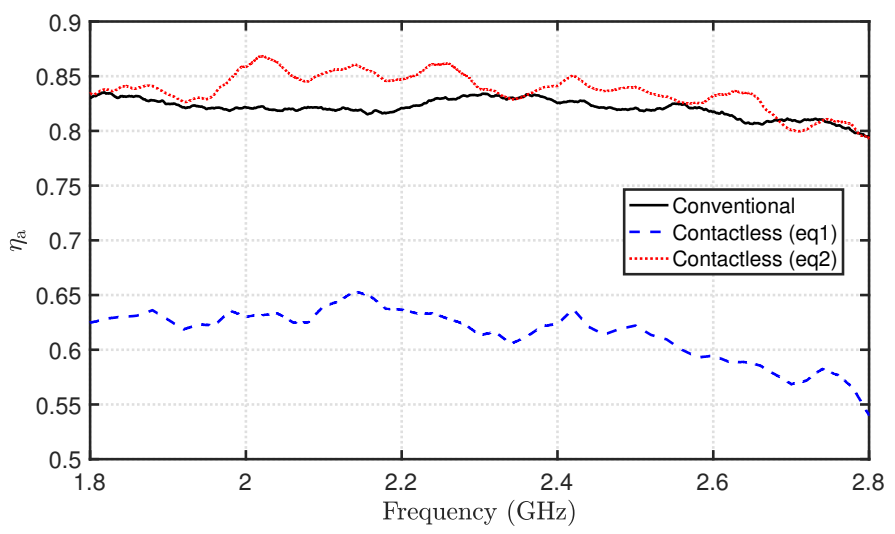

Fig. 5. Radiation efficiency of the patch antenna measured in the ESYCOM $\mathrm{RC}$ using the invasive and the contactless approach, as a function of frequency. Results are smoothed over a $30 \mathrm{MHz}$ window.

RC $Q$-factors for both load conditions are computed in the time domain using the transmission coefficient $S_{21}$ between both measurement antennas (A1 and A2) by fitting the power delay profile $\operatorname{PDP}=\left\langle\left|\operatorname{IFT}\left(S_{21}\right)\right|^{2}\right\rangle[7]$ where the average denoted $\langle$.$\rangle is calculated over all the configurations in addition$ to a sliding frequency window of $200 \mathrm{MHz}$. As the two $Q$-factors are very close, we present also $\Delta Q^{-1} / \overline{Q^{-1}}=$ $2\left(Q_{50}^{-1}-Q_{\mathrm{OC}}^{-1}\right) /\left(Q_{50}^{-1}+Q_{\mathrm{OC}}^{-1}\right)$ in Fig. 4 as a function of frequency. This quantity is always positive, meaning that $Q_{\mathrm{OC}}^{-1}<Q_{50}^{-1}$ for all frequencies. This is coherent as more losses are expected within the RC when the AUT is connected to the $50 \Omega$ load. Also, it decreases as a function of frequency, which is expected as the contribution of the AUT on the RC $Q$-factor becomes smaller.

\section{B. Results}

The radiation efficiency measured using the contactless setup is presented in Fig. 5 using eq1 (7) and eq2 (8) as a function of frequency. To evaluate the radiation efficiency accuracy, results are compared to those obtained through a conventional invasive measurement method within the same $\mathrm{RC}$ (two-antenna approach in [7]). 
TABLE I

Radiation EFFiciency Mean Value and Standard DeViation over THE FREQUENCY RANGE FOR THE EXPERIMENTS CONDUCTED IN THE $19 \mathrm{M}^{3}$ ReVERBERATION CHAMBER.

\begin{tabular}{|l|c|c|}
\hline & Mean & Standard Deviation \\
\hline Conventional & $81.9 \%$ & 0.0102 \\
\hline Contactless eq1 & $61.6 \%$ & 0.0228 \\
\hline Contactless eq2 & $83.7 \%$ & 0.0163 \\
\hline Contactless eq2 (ideally matched AUT) & $82.3 \%$ & 0.0149 \\
\hline Contactless eq2 (ideal loads) & $78.6 \%$ & 0.0131 \\
\hline
\end{tabular}

This method is chosen as a reference as it is widely used and well known to provide accurate and repeatable radiation efficiency estimations within an RC [30], [31].

These results show that the newly introduced method using (8), i.e., eq2, allows obtaining very similar results to the ones obtained using a conventional invasive RC approach applied in the same measurement conditions (same RC $Q$-factor and same stirring processes). Indeed, the averaged relative error is equal to only $2.3 \%$.

As the estimated efficiency using the conventional method is almost constant over the frequency range, we compute the mean and the standard deviation of the three estimated efficiencies for comparison purposes; they are presented in Table II The radiation efficiency averaged over the whole frequency band is equal to $81.9 \%$ with the conventional method and $83.7 \%$ with the contactless approach in the case of eq2. However, the standard deviation is higher for the contactless approach.

Indeed, the proposed method is based on the estimation of a small $Q$-factor variation due to the AUT load modification and is therefore more sensitive to the non-ideal behavior of the $\mathrm{RC}$, leading to higher fluctuations as a function of frequency.

It has to be noted that, as expected, the contactless approach using (7), i.e., eq1, leads to a strongly underestimated radiation efficiency (61.6\% on average over the frequency bandwidth). This is due to the fact that (7) does not take into account that a part of the power arising on the AUT is actually reflecting back into the RC (due to mismatches). This power is thus considered as absorbed by the AUT, which artificially decreases its radiation efficiency. These results demonstrate the possibility to estimate the antenna radiation efficiency using a contactless approach, i.e., without connecting the AUT to the VNA, by measuring the RC $Q$-factor for two AUT load conditions. Also, it confirms that the antenna $Q$-factor formula introduced in [29] better models the antenna absorption within an RC.

\section{Reflection coefficient approximations}

The contactless method requires the determination of $\left|\Gamma_{\mathrm{a}, \mathrm{L} x}\right|$ from (5) and thus the measurement of both reflection coefficients $\Gamma_{\mathrm{a}}$ and $\Gamma_{\mathrm{L} x}$. In this paper, they are measured directly using a VNA, i.e., using an invasive measurement. Although the impedance is usually less sensitive than radiation properties to the proximity of cables, impedance measurements might not be possible from a practical point of view, especially if the AUT is specifically designed including an integrated miniaturized switching device. Thus, we evaluate

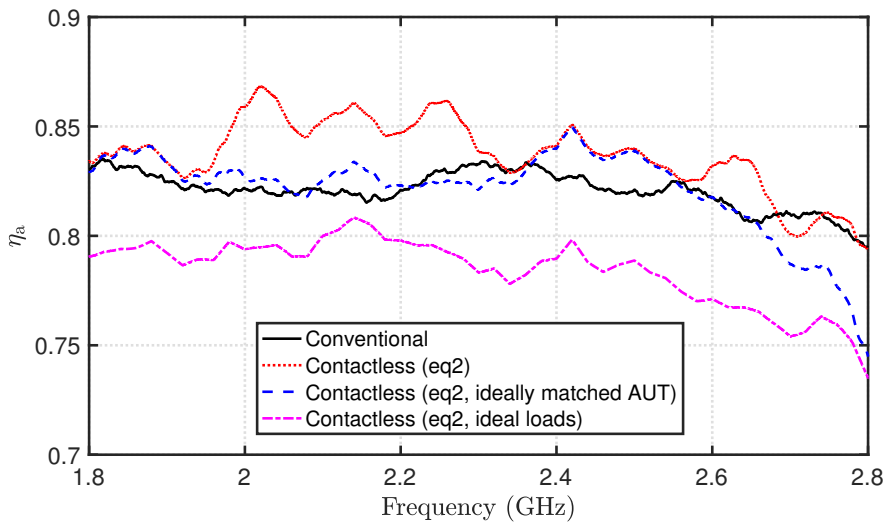

Fig. 6. AUT radiation efficiency as a function of frequency, measured using the contactless approach considering different approximations regarding the reflection coefficients.

in the following the impact of no a priori knowledge on these reflection coefficients. Accordingly, two approximations are successively done: (1) the AUT is assumed to be ideally matched to $50 \Omega$ so that $\Gamma_{\mathrm{a}}=0$ and thus $\left|\Gamma_{\mathrm{a}, \mathrm{L} x}\right|=\left|\Gamma_{\mathrm{L} x}\right|$, and (2) we consider an ideal OC so that $\Gamma_{\mathrm{OC}}=1$ and thus $\left|\Gamma_{\mathrm{a}, \mathrm{OC}}\right|=1$ and an ideal $50 \Omega$ load so that $\Gamma_{50}=0$ and thus $\left|\Gamma_{\mathrm{a}, 50}\right|=\left|\Gamma_{\mathrm{a}}\right|$. Radiation efficiencies obtained from these two approximations are presented in Fig. 6 and compared with the conventional method and the contactless one without approximation. Considering the AUT ideally matched to $50 \Omega$ leads to very similar results to the ones without approximation (the average radiation efficiency is equal to $82.3 \%$ ) which is due to the fact that the AUT is well-matched in this frequency band (see Fig. 3). Indeed, when $\Gamma_{\mathrm{a}}$ is very low (around $1.85 \mathrm{GHz}$ and $2.5 \mathrm{GHz}$ ), the two curves merge. However, if we consider ideal loads, a systematic underestimation of the radiation efficiency is observed (the average radiation efficiency is equal to $78.6 \%$ ). This is mainly due to the switch and the cable insertion losses located between the calibration port and the load, which are not taken into account and thus, not properly compensated.

\section{Stirring effect}

This part is dedicated to the evaluation of the measurement uncertainties according to the stirring process. All results shown in the previous parts were obtained by applying all the stirring possibilities of the measurement setup, including the mechanical stirring (72 configurations), the source stirring (17 configurations) and the AUT stirring (4 configurations), leading up to 4896 configurations. The objective of such stirring process is to lower the measurement uncertainties, i.e., to lower the standard deviation of the radiation efficiency estimation. However, the accuracy of any RC measurements relies on the effective sample size generated by the stirring process, i.e., the number of uncorrelated configurations. A correlation coefficient $\rho$ is first calculated using the 72 stirrer positions for a single source position and a single AUT position, based on the transmission coefficient between the two measurement antennas. The effective sample size $N_{\text {eff }}$ is then deduced from $\rho[33$. Since it presents fluctuations as a 
TABLE II

NORMALIZED STANDARD DEVIATIONS OF THE ESTIMATED RADIATION EFFiciency ACCORDING to the Mechanical StirRing Process.

\begin{tabular}{|l|c|}
\hline & Normalized Standard Deviation \\
\hline Prediction [34] & 0.184 \\
\hline Source stirring & 0.173 \\
\hline AUT stirring & 0.186 \\
\hline
\end{tabular}

function of frequency, its value is averaged in the $2 \mathrm{GHz}$ to $2.4 \mathrm{GHz}$ frequency band. This estimation is made for each source and AUT position before being averaged and leads to $N_{\text {eff }}=59$. It has to be noted that $N_{\text {eff }}$ has been computed for the two AUT load conditions but it leads to very similar results thus, only the results obtained for the OC case are presented thereafter.

Once the effective sample size is known, it is possible to evaluate the normalized standard deviation of the radiation efficiency estimation as [34]:

$$
\frac{\sigma_{\eta_{\mathrm{a}}}}{\left\langle\eta_{\mathrm{a}}\right\rangle} \approx \sqrt{\frac{2}{N_{\mathrm{eff}}}} .
$$

In the present case $\left(N_{\mathrm{eff}}=59\right)$, the normalized standard deviation is estimated to be equal to 0.184 . In order to validate this result, we now aim at evaluating the normalized standard deviation of the radiation efficiency estimation from the measurement results. As two types of stirring, namely the source stirring and the AUT stirring, are performed in addition to the conventional mechanical stirring, two different estimations of the normalized standard deviation are computed: one using the source stirring and the other one using the AUT stirring. First, we estimate $\sigma_{\eta_{\mathrm{a}}}^{p_{\text {src }}}$ from all source positions and for one AUT position. The result is then averaged over all AUT positions $\left\langle\sigma_{\eta_{\mathrm{a}}}^{p_{\text {src }}}\right\rangle_{p_{\mathrm{AUT}}}$. The same process is made considering only the 4 AUT positions for one source position, before averaging

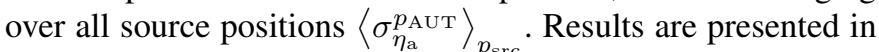
Table II Both estimations from the measurement results are in very good agreement with the theoretical prediction (relative error of $6 \%$ for the source stirring estimation and $1 \%$ for the AUT stirring).

This confirms that the normalized standard deviation can be predicted directly from $N_{\text {eff. In the present case, we evaluated }}$ 10 uncorrelated source positions out of the 17 using the same process than the one used for the mechanical stirring. If we consider that the 4 AUT positions are uncorrelated (4 positions being too low to accurately estimate the effective sample size), it comes $4 \times 10 \times 59=2360$ uncorrelated configurations. According to 9 , this leads to a predicted normalized standard deviation that is equal to 0.029 .

\section{VALIDATION IN A SMALL RC}

In this section, we aim at validating the proposed measurement method using a much smaller RC, thus exhibiting a much smaller $Q$-factor. These experiments have been conducted in the $1 \mathrm{~m}^{3} \mathrm{RC}$ of the Institut Langevin with the experimental setup presented in Fig. 7 The AUT is the same wideband patch antenna as in the previous section. The $Q$-factor being much smaller than in the large RC (about 1700 at the central

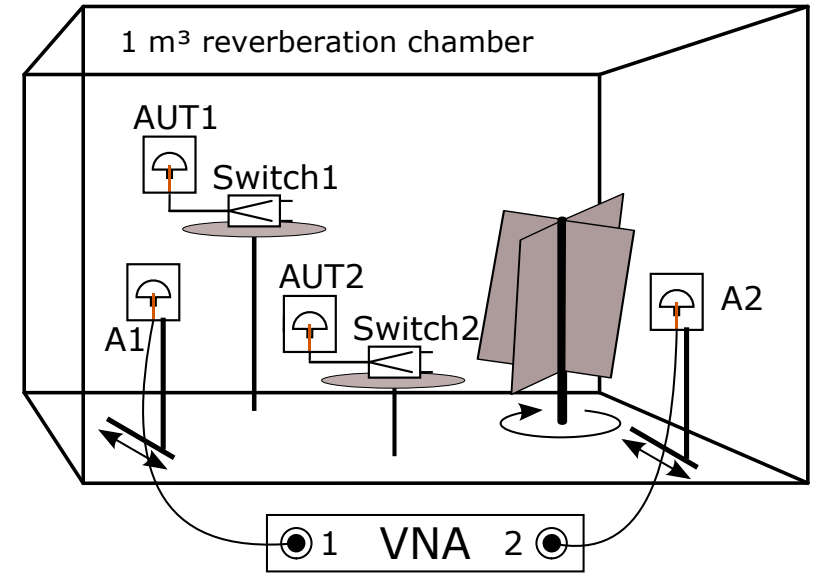

Fig. 7. Contactless antenna efficiency measurement setup in the small RC. The vertical mode stirrer is rotating around its axis and both measurement antennas A1 and A2 are translated along linear stages.

frequency), two identical AUTs have been placed within the $\mathrm{RC}$ in order to enhance the AUT absorption strength so $N=2$ in (8). They are both connected to their own mechanical switch (that can switch between an OC and a $50 \Omega$ load) through a $30-\mathrm{cm}$ coaxial cable. For practical reasons (especially the limited size of the RC), the two antennas A1 and A2 are the same as the AUTs so that four identical patch antennas are used: two as AUTs and two as measurement antennas (A1 and A2). Scattering parameters are measured thanks to an Anritsu VNA in the same frequency range $(1.8 \mathrm{GHz}$ to $2.8 \mathrm{GHz})$ with 10001 frequency points. A1 and A2 antennas are positioned on a translation stage in order to perform source stirring over 9 configurations ( 3 positions for each antenna) in addition to the mechanical rotating stirrer that provides 72 positions. Thus, all results are averaged over 648 configurations.

The same procedure as in the previous section is conducted here regarding the AUT and the load impedances reflection coefficients measurement, the results are not presented for brevity. $Q$-factors are presented in Fig. 8 as a function of frequency. As expected, $Q$ increases with frequency, and it is slighty lower for the $50 \Omega$ case as more energy is absorbed by this load. In order to highlight the sensitivity of the $Q$ factor estimation, we also computed the $Q$-factor for the empty case, i.e. when the AUTs are removed, whereas everything else, including the cables and the switches, are kept within the RC. We can see that this result is not consistent with the previous ones. Indeed, due to the estimation fluctuations, $Q$ in the empty case is sometimes higher and sometimes lower than the one with AUT connected to the OC. This is precisely why we suggested in this approach the use of two load conditions obtained without manual manipulation and no empty RC measurement.

The radiation efficiency is measured using the conventional setup as well as with the contactless setup and results are presented in Fig. 9 as a function of frequency. The values of $\left|\Gamma_{\mathrm{a}, \mathrm{L} x}\right|$ have been computed by averaging the measurements performed on the 2 switches. Mean values as well as standard deviations over the frequency range for each estimation are presented in Table III These results confirm those obtained in 


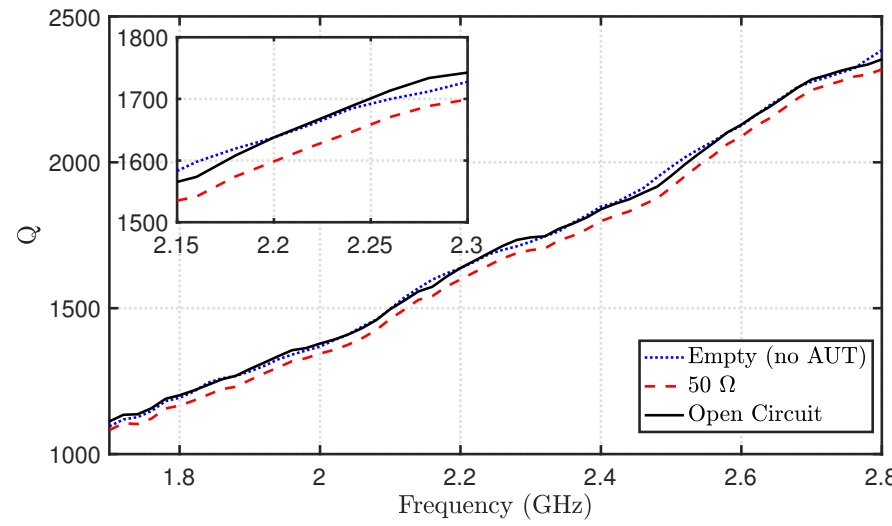

Fig. 8. RC $Q$-factors of the small $\mathrm{RC}$ as a function of frequency, measured without any AUT (blue) or when both AUTs are connected to an OC (black) and a $50 \Omega$ load (red).

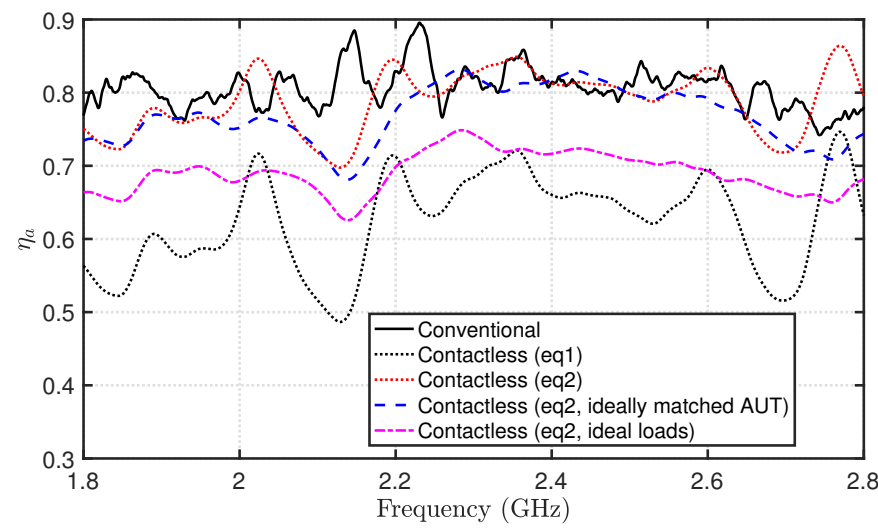

Fig. 9. AUT radiation efficiency as a function of frequency, measured in the small RC using the invasive and the contactless approaches, considering different approximations regarding the reflection coefficients. The results are smoothed over a $30 \mathrm{MHz}$ window.

section III-B a very similar radiation efficiency estimation is obtained between the conventional and the contactless (eq2) methods $(2 \%$ absolute difference on average, and a relative difference of $6 \%$ on average) although the standar deviation as a function of frequency is higher for the contactless case. It has to be noted that the mean number of uncorrelated stirrer positions, between $2 \mathrm{GHz}$ and $2.4 \mathrm{GHz}$, is evaluated to be 21 , for a mean number of uncorrelated measurement antenna configurations of 5.4. This leads to 113 uncorrelated samples, which is much smaller than the 2360 uncorrelated samples of the ESYCOM RC; this explains why the standard deviations are larger than the ones obtained previously. Again, the radiation efficiency estimated through eq1 is not consistent with the one obtained using the conventional method. Also, the same approximations as the ones made in $\mathrm{III}-\mathrm{C}$ regarding the reflection coefficients are made. Considering the AUT perfectly matched to $50 \Omega$ leads to a slight underestimation of only $2 \%$ on average whereas considering ideal loads leads to a strong underestimation of about $10 \%$.

\section{CONClusion And Discussion}

In this paper, we introduced an original measurement method to evaluate the antenna radiation efficiency within an
TABLE III

Radiation EFFiciency Mean Value and Standard Deviation over THE FREQUENCY RANGE FOR THE EXPERIMENTS CONDUCTED IN THE $1 \mathrm{M}^{3}$ Reverberation Chamber.

\begin{tabular}{|l|c|c|}
\hline & Mean & Standard Deviation \\
\hline Conventional & $80.9 \%$ & 0.0254 \\
\hline Contactless eq2 & $78.9 \%$ & 0.0424 \\
\hline Contactless eq2 (ideally matched AUT) & $76.9 \%$ & 0.0387 \\
\hline Contactless eq2 (ideal loads) & $69.0 \%$ & 0.0278 \\
\hline
\end{tabular}

RC. It is contactless so that the method does not require to connect the AUT to an analyzer. It is based on the measurement of the RC $Q$-factor for two load conditions of the AUT. It is emphasized that the two measurements are performed without modifying the measurement setup (no manual handling) thanks to remotely-controlled mechanical switches. An OC and a $50 \Omega$ load have been considered here as the two load impedances in order to enhance the measurement sensitivity. It has been validated with a wideband patch antenna in the $1.8 \mathrm{GHz}$ to $2.8 \mathrm{GHz}$ frequency range in two different RCs of different volumes: $1 \mathrm{~m}^{3}$ and $19 \mathrm{~m}^{3}$. The retrieved radiation efficiency is very similar to the one obtained using a conventional invasive approach [7] (average relative difference of $2.3 \%$ in the large $\mathrm{RC}$ and $6 \%$ in the small one).

This method paves the way for non-invasive antenna radiation efficiency measurement, which is highly suitable for electrically small antennas as an invasive setup disturbs their impedance and radiation properties. However, this method also exhibits limitations. The contactless approach is based on the estimation of the RC $Q$-factor variation for two AUT load conditions. Depending of the considered setup (AUT, loads, and RC $Q$-factor), this variation may be small. Therefore, the contactless method is more sensitive to the nonideal behavior of the RC and its stirring capabilities than conventional measurements. This has been seen in the second set of measurement (Section IV) in an RC with a smaller $Q$-factor: the retrieved efficiency exhibits higher fluctuations over the frequency range than in the RC of higher $Q$-factor. This limitation can be counterbalance by performing additional stirring as well as enhancing the number of AUTs (increasing $N$ ) in order to raise the RC $Q$-factor variation.

In this paper, we also confirmed that the antenna $Q$-factor formulation introduced in 2018 [29] describes the absorption introduced by an antenna within an RC in a better manner than the former model introduced by D. Hill in 1998 [28]. Indeed, some of the energy reflected back by the antenna load towards the RC was not taken into account by the original model, leading to a strong radiation efficiency underestimation once using this model in our method.

Future works will include miniaturization of the switching device in order to integrate it into the AUT. Specific antenna design including the switching device could be realized for testing purposes only.

\section{REFERENCES}

[1] C. A. Balanis, Antenna theory: analysis and design, Wiley-Interscience, USA, 2005. 
[2] P. Hallbjorner, Reflective antenna efficiency measurements in reverberation chambers, Microwave Opt. Technol. Lett., vol. 30, no. 5, pp. 332335, Sept 2001.

[3] M. Piette, Antenna radiation efficiency measurements in a reverberation chamber, Asia-Pacific Radio Sci. Conf., 2004, pp. 19-22, doi: 10.1109/APRASC.2004.1422388.

[4] K. Rosengren and P.-S. Kildal, Radiation efficiency, correlation, diversity gain and capacity of a six-monopole antenna array for a MIMO system: theory, simulation and measurement in reverberation chamber, IEE Proc. Microw. Antennas Propag., vol. 152, no. 1, Feb. 2005.

[5] P. Besnier, J. Sol, A. Presse, C. Lemoine and A. -C. Tarot, Antenna efficiency measurement from quality factor estimation in reverberation chamber, Europ. Microw. Conf. (EuMC), 2016, pp. 715-718, doi: 10.1109/EuMC.2016.7824443.

[6] W. Krouka, F. Sarrazin, and E. Richalot, Influence of the reverberation chamber on antenna characterization performances, Int. Symp. Electromagn. Compat. (EMC EUROPE), Aug 2018, pp. $329 ? 333$.

[7] C. L. Holloway, H. A. Shah, R. J. Pirkl, W. F. Young, D. A. Hill, and J. Ladbury, Reverberation chamber techniques for determining the radiation and total efficiency of antennas, IEEE Trans. Antennas Propag., vol. 60, no. 4, pp. 1758?1770, Apr 2012.

[8] W. Krouka, F. Sarrazin, J. Sol, P. Besnier and E. Richalot, Comparison of antenna radiation efficiency measurement techniques in reverberation chamber using or not a reference antenna, Europ. Conf. Antennas Propag. (EuCAP), Copenhagen, Denmark, 2020, pp. 1-4.

[9] J. DeMarinis, The antenna cable as a source of error in EMI measurements, IEEE Int. Symp. Electromagn. Compat., 1988, pp. 9-14, doi: 10.1109/ISEMC.1988.14078.

[10] S. Saario, D. V. Thiel, J. W. Lu and S. G. O'Keefe, An assessment of cable radiation effects on mobile communications antenna measurements, IEEE Int. Symp. Antennas Propag. Soc., Digest, 1997, pp. 550553 vol.1, doi: 10.1109/APS.1997.630220.

[11] C. Icheln, J. Ollikainen, and P. Vainikainen, Reducing the influence of feed cables on small antenna measurements, Electron. Lett., vol. 35, no. 15, pp. 1212?1214, July 1999.

[12] L. Liu, Y. F. Weng, S. W. Cheung, T. I. Yuk and L. J. Foged, Modeling of cable for measurements of small monopole antennas, Loughborough Antennas Propag. Conf., 2011, pp. 1-4, doi: 10.1109/LAPC.2011.6114153.

[13] O. Staub, J.-F. Zrcher and A. Skrivervik, Some considerations on the correct measurement of the gain and bandwidth of electrically small antennas, Microw. Opt. Technol. Lett., 17, 156-160, 1998 , https://doi.org/10.1002/(SICI)1098-2760(19980220)17:3;156::AIDMOP2 $\_3.0 . C O ; 2-I$.

[14] L. Huitema, C. Delaveaud and R. D'Errico, Impedance and radiation measurement methodology for ultra miniature antennas, IEEE Trans. Antennas Propag., vol. 62, no. 7, pp. 3463-3473, July 2014.

[15] K. S. Leong, M. L. Ng and P. H. Cole, Investigation of RF cable effect on RFID tag antenna impedance measurement, IEEE Int. Symp. Antennas Propag. Soc., 2007, pp. 573-576, doi: 10.1109/APS.2007.4395558.

[16] T. Fukasawa, N. Yoneda and H. Miyashita, Investigation on Current Reduction Effects of Baluns for Measurement of a Small Antenna, IEEE Trans. Antennas Propag., vol. 67, no. 7, pp. 4323-4329, July 2019, doi: 10.1109/TAP.2019.2911360.

[17] C. Icheln, M. Popov, P. Vainikainen and S. He, Optimal reduction of the influence of $R F$ feed cables in small antenna measurements, Microw. Opt. Tech. Lett., vol. 25, pp. 194-196, 2000, doi:10.1002/(SICI)10982760(20000505)25:33.0.CO;2-\#.

[18] J. L. Araque Quijano, L. Scialacqua, J. Zackrisson, L. J. Foged, M. Sabbadini and G. Vecchi, Suppression of Undesired Radiated Fields Based on Equivalent Currents Reconstruction From Measured Data, IEEE Antennas Wireless Propag. Lett., vol. 10, pp. 314-317, 2011, doi: 10.1109/LAWP.2011.2135831.

[19] R. R. Lao, W. Liang, Yung-Sheng Chen and J. H. Tarng, The use of electro-optical link to reduce the influence of RF cables in antenna measurement, IEEE Int. Symp. Microw., Antenna, Propag. EMC Tech. Wireless Comm., 2005, pp. 427-430, vol. 1, doi 10.1109/MAPE.2005.1617940.

[20] M. Hachemi, S. Bories, K. H. Khlifa and C. Delaveaud, Miniature antenna radiation pattern measurement using fiber-optic link, Proc. 4th Europ. Conf. Antennas Propag., Barcelona, Spain, 2010, pp. 1-5.

[21] J. Appel-Hansen, Accurate determination of gain and radiation patterns by radar cross-section measurements, IEEE Trans. Antennas Propag., vol. 27, no. 5, pp. 640-646, Sep. 1979, doi: 10.1109/TAP.1979.1142156.

[22] W. Wiesbeck and E. Heidrich, Wide-band multiport antenna characterization by polarimetric RCS measurements, IEEE Trans. Antennas Propag., vol. 46, no. 3, pp. 341-350, March 1998, doi: 10.1109/8.662653.
[23] A. Reis, F. Sarrazin, P. Besnier, P. Pouliguen and E. Richalot, Antenna radar cross section measurement within mode-stirred reverberation chamber, 15th Europ. Conf. Antennas Propag. (EuCAP), 2021, pp. 1-2, doi: 10.23919/EuCAP51087.2021.9410961.

[24] A. Reis et al., Radar cross-section pattern measurements in a modestirred reverberation chamber: Theory and experiments, IEEE Trans. Antennas Propag., doi: 10.1109/TAP.2021.3060581.

[25] A. Reis, F. Sarrazin, P. Besnier, P. Pouliguen and E. Richalot, Contactless Antenna Gain Pattern Estimation From Backscattering Coefficient Measurement Performed Within Reverberation Chambers, IEEE Trans. Antennas Propag., doi: 10.1109/TAP.2021.3111184.

[26] U. Carlberg, P. S. Kildal, A. Wolfgang, O. Sotoudeh and C. Orlenius, Calculated and measured absorption cross sections of lossy objects in reverberation chamber, IEEE Trans. Electromagn. Compat., vol. 46, no. 2 , pp. 146-154, May 2004

[27] G. Lerosey and J. de Rosny, Scattering cross section measurement in reverberation chamber, IEEE Trans. Electromagn. Compat., vol. 49, no. 2, pp. 280-284, May 2007.

[28] D. Hill, Electromagnetic theory of reverberation chambers, National Institute of Standards and Technology, Gaithersburg, MA, USA, Tech Rep. 1506, 1998

[29] A. Cozza, Power loss in reverberation chambers by antennas and receivers, IEEE Trans. Electromagn. Compat., vol. 60, no. 6, pp. 20412044, Dec. 2018.

[30] A. Hubrechsen et al., The Effect of Noise on Reverberation-Chamber Measurements of Antenna Efficiency, IEEE Trans. Antennas Propag., doi: 10.1109/TAP.2021.3083822.

[31] L. A. Bronckers, K. A. Remley, B. F. Jamroz, A. Roc?h and A. Bart Smolders, Uncertainty in Reverberation-Chamber AntennaEfficiency Measurements in the Presence of a Phantom, IEEE Trans. Antennas Propag., vol. 68, no. 6, pp. 4904-4915, June 2020, doi: 10.1109/TAP.2020.2969883.

[32] K. Kurokawa, Power waves and the scattering matrix, IEEE Trans. Microwave Theory Tech., vol. 13, no. 2, pp. 194-202, March 1965, doi: 10.1109/TMTT.1965.1125964.

[33] C. Lemoine, P. Besnier and M. Drissi, Estimating the effective sample size to select independent measurements in a reverberation chamber, IEEE Trans. Electromagn. Compat., vol. 50, no. 2, pp. 227-236, May 2008, doi: 10.1109/TEMC.2008.919037.

[34] X. Chen, On statistics of the measured antenna efficiency in a reverberation chamber, IEEE Trans. Antennas Propag., vol. 61, no. 11, pp. 5417-5424, Nov. 2013, doi: 10.1109/TAP.2013.2276920. 